\title{
Effect of weak magnetic fields treatment on photoluminescence of III-V single crystals
}

\author{
R.V. Konakova, S.M. Red'ko, V.V. Milenin, R.A. Red'ko \\ V. Lashkaryov Institute of Semiconductor Physics, National Academy of Sciences of Ukraine, \\ 45, prospect Nauky, 03028 Kyiv, Ukraine \\ Phone:+38 (044) 525-94-64; fax:+38 (044) 525-61-82,e-mail: redko.rom@gmail.com
}

\begin{abstract}
The long-term transformations of photoluminescence of GaP, GaAs and InP single crystals treated with pulsed weak magnetic fields are obtained. The treatments were performed in two regimes, namely, single-pulse $(\tau=30 \mathrm{~ms})$ and multi-pulse $(\tau=$ $1.2 \mathrm{~ms}$ ) ones, at varying magnitudes of magnetic induction. The defect structure transformations were inferred from the radiative recombination spectra in the $0.6-2.5 \mu \mathrm{m}$ at $77 \mathrm{~K}$. A possible mechanism of observed modifications related to the electron spin transformation is discussed.
\end{abstract}

Keywords: photoluminescence, weak magnetic field, impurity-defect composition.

Manuscript received 02.12.13; revised version received 29.01.14; accepted for publication 20.03.14; published online 31.03.14.

\section{Introduction}

Weak magnetic fields (WMF) induce modification of the defect subsystem in solids in some nontrivial way. This method of physical action is of interest and promising from scientific point of view as well as important practically [1-4]. The energy absorbed as a result of treatment in such magnetic fields is three orders of magnitude smaller than the thermal energy of electrons at room temperature, which complicates the explanation of the observed effects. However, even this energy action can cause both reversible and irreversible changes in the defect subsystems of solids [1]. Despite numerous investigations in this field and some proposed explanations, the problem of WMF interaction with practically important III-V semiconductors still remains open [3]. Therefore, search for new effects related to the WMF treatment of substances (in particular, semiconductors) is urgent. The results to be obtained would be of use for better understanding of the mechanisms of defect subsystem modification caused by low-energy influence.

Gallium phosphide is one of the best-understood semiconductors with the indirect energy gap. Its comparatively big value $E_{g}=2.24 \mathrm{eV}$ (at $300 \mathrm{~K}$ ) leads to predominant light emission in the visible spectral range. GaAs and InP are important for creation of solid state devices such as solar cells, lasers, Schottky diodes, as well as substrate materials. That is why these prototypical III-V compounds were the objects of our investigation. Although the properties of such semiconductors have been studied in detail, the problem of mechanisms of their interaction with WMF still remains open.

\section{Experimental}

All the III-V samples under investigation were Czochralski-grown with orientation (111) and $n$-type doped with Te. Their characteristics are presented in 
Table. Impurity-defect luminescence is sensitive to impurities and imperfections, especially in the best understood III-V compounds under study. Therefore, the defect structure of our samples was studied using the photoluminescence (PL) method. The PL spectra of semiconductors were measured in the wavelength range 600 to $2000 \mathrm{~nm}$ at $77 \mathrm{~K}$ under excitation by the PZh-100 lamp radiation $\left(\lambda_{\mathrm{ex}} \leq 600 \mathrm{~nm}\right)$ passing through the crossing filters. The PL data were normalized to the initial value of band intensity.

Three groups of samples were treated in the single-pulse regime (I) of WMF with different values of $B: 8,15$ and $60 \mathrm{mT}$. The duration of WMF pulse in this case was $\tau_{I}=30 \mathrm{~ms}$. The multi-pulse regime (II) with parameters $B=60 \mathrm{mT}, f=10 \mathrm{~Hz}, \tau_{I I}=1.2 \mathrm{~ms}, t=$ $60 \mathrm{~s}$ was the second type of applied treatment. The specimen receiving no WMF treatment was used as the reference one.

\section{Results and discussion}

Fig. 1 illustrates the initial state of III-V semiconductor samples. The GaAs spectrum at $77 \mathrm{~K}$ exhibits two wellresolved luminescence bands, at 1.02 and $1.21 \mathrm{eV}$ (Fig. 1). The first peak is attributed to $\mathrm{V}_{\mathrm{Ga}} \mathrm{V}_{\mathrm{As}}$ or $\mathrm{Cu}_{\mathrm{Ga}} \mathrm{V}_{\mathrm{As}}$, and the second one is assigned to $\mathrm{V}_{\mathrm{Ga}} \mathrm{Te}_{\mathrm{As}}$ complexes [5].

Table. Characteristics of the materials studied.

\begin{tabular}{|c|c|c|}
\hline Material & $\begin{array}{c}\text { Concentration of } \\
\text { charge carriers, } \mathrm{cm}^{-3}\end{array}$ & $\begin{array}{c}\text { Thickness, } \\
\mu \mathrm{m}\end{array}$ \\
\hline $\mathrm{GaP}$ & $8 \times 10^{16}$ & 350 \\
\hline $\mathrm{GaAs}$ & $(1 \ldots 5) \times 10^{16}$ & 400 \\
\hline $\mathrm{InP}$ & $1 \times 10^{18}$ & $250 \ldots 300$ \\
\hline
\end{tabular}

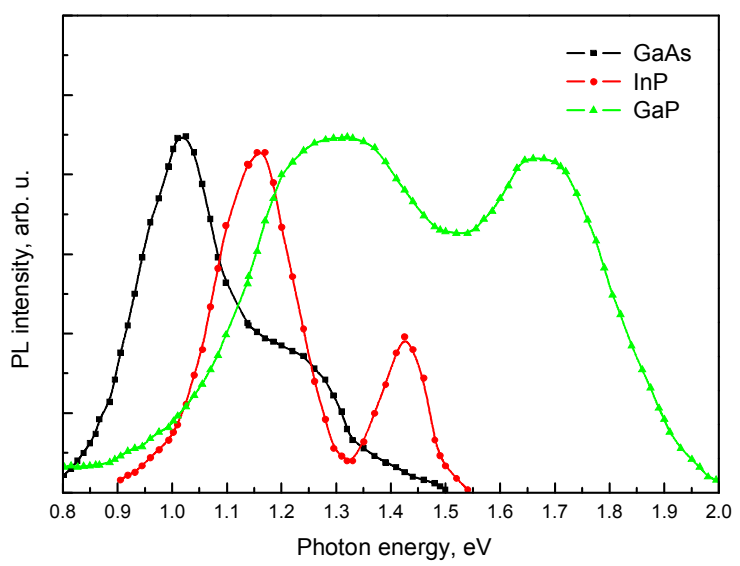

Fig. 1. PL spectra at $77 \mathrm{~K}$ of the initial state of the samples studied before WMF treatment.
The radiative recombination spectrum of $\mathrm{InP}$ crystals consists of two bands peaked at about 1.14 and $1.41 \mathrm{eV}$ (Fig. 1) that are attributed to $\mathrm{V}_{\mathrm{P}} \mathrm{Fe}_{\mathrm{In}}$ or $\mathrm{Si}_{\mathrm{P}} \mathrm{Fe}_{\text {In }}$ [6] and band-to-band or shallow donor recombination [7], respectively. In our case, the edge origin of the latter band is more probable because of well fitting by the Varshni's approach. The decrease of the bandgap of indium phosphide with increase of temperature (primarily due to the electron-phonon interaction) results in the redshift of peak position. It could be fitted by the well-known formula [8] (Fig. 2):

$E_{g}(T)=E_{g}(0)-\frac{\alpha T^{2}}{T+\beta}$

with empirical coefficients $E_{g}(0)=1.415 \mathrm{eV}, \alpha=$ $3.5 \cdot 10^{-4} \mathrm{eV} / \mathrm{K}, \beta=215 \mathrm{~K}$. The PL spectrum in the red region of $\mathrm{GaP}$ crystals contains two bands near 1.7 and $1.3 \mathrm{eV}$ at $77 \mathrm{~K}$ (Fig. 1). At room temperature, these peaks redshift to 1.5 and $1.2 \mathrm{eV}$, respectively. The origin of the observed bands is ascertained as being based on the analysis of their half-widths $(0.20 \ldots 0.35 \mathrm{eV})$. The first band may be associated with low tellurium concentration [9] (for example, with $\mathrm{Te}$ (donor)$\left[\mathrm{V}_{\mathrm{Ga}}+2 \mathrm{Te}_{\mathrm{P}}\right]$ (acceptor) or $\mathrm{Cu}_{\mathrm{Ga}}+\mathrm{Te}_{\mathrm{P}}$ complex). The second peak corresponds to $\mathrm{O}$ impurity [10]. Band-toband emission for $\mathrm{GaP}$ samples is out of the measured spectral interval, so it was not observed.
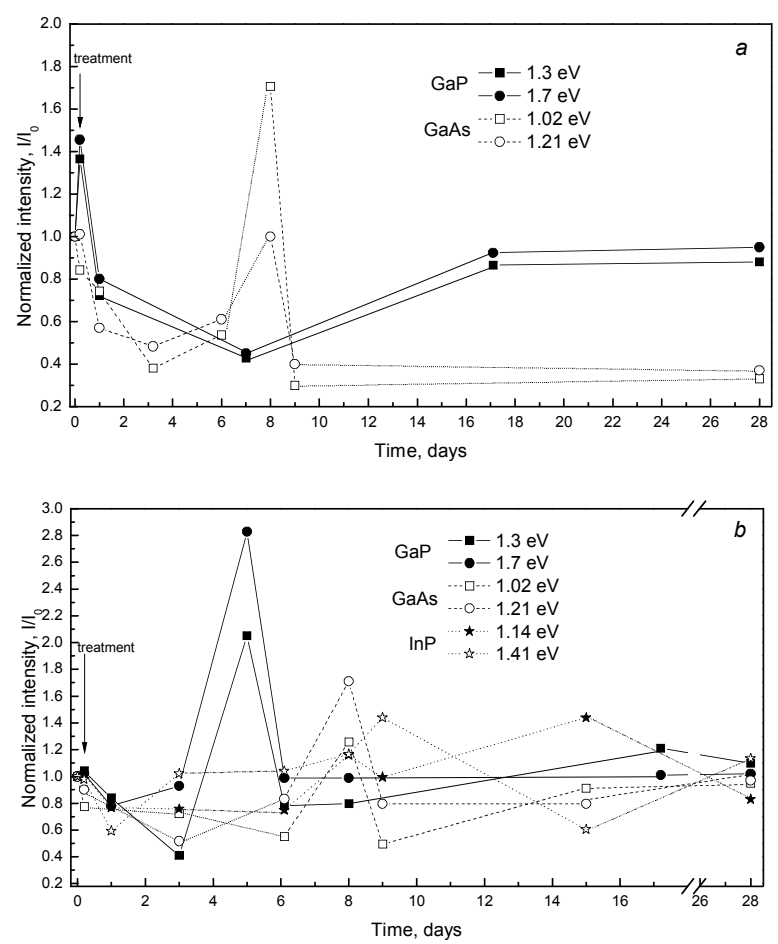

Fig. 2. Intensity of PL bands (at $77 \mathrm{~K}$ ) of III-V crystals as a function of time after MF treatment (regime I) with induction $B_{1}=8 \mathrm{mT}$ (a) and $B_{3}=60 \mathrm{mT} \mathrm{(b).}$ 
To reveal the features of the influence of WMF treatment on the defect structure of III-V compounds, we considered the changes of the characteristics of PL bands after $\sim 30$ days of storage in detail. The PL measurements were carried out every day, but for more convenient analyzing, only informative points were accepted to plot time dependences. The influence of single pulse (regime I) at different values of magnetic induction ( 8 and $60 \mathrm{mT}$ ) on the PL in these semiconductors is shown in Fig. $2 \mathrm{a}$ and $2 \mathrm{~b}$. The increase of magnetic induction up to $B_{2}=15 \mathrm{mT}$ results in qualitatively similar changes in radiative recombination (nonmonotonic) of materials studied but with some distinctions in the intensity values. Therefore, these data do not need special attention and are not presented in Figures. Exposure to MF in the regime I resulted in decrease of the intensity of almost all observed bands (Fig. 2a). The behavior of PL bands is oscillatory with attenuation to some steady state. One can conclude that the changes of the intensity of radiative recombination in all III-V semiconductors are nonmonotonic and do not cease when the treatment ends. WMF-induced transformations of spectra occur during $20 . .60$ days for different samples. Some steady state was reached after this period. It should be noted that for all semiconductors studied, the most efficient reactions were observed for donor-acceptor (DA) complexes that consisted of the vacancies of matrix material. This fact is of importance for subsequent discussion.

One can note that the emission intensity of astreated $\mathrm{GaP}$ samples increases (Fig. 2a) but for GaAs does not change essentially. The subsequent behavior of all the observed bands is similar: the intensity of radiative recombination decreases with time up to 8 days. The maximum of emission for GaAs and the minimum for $\mathrm{GaP}$ are observed during this period.

The pulse of MF with $B_{3}=60 \mathrm{mT}$ results in other features. The dependences of PL intensity on the duration of WMF treatment of GaP, GaAs and InP that contained the bands peaking at $1.3,1.7 ; 1.02,1.21$ and 1.14, $1.41 \mathrm{eV}$, respectively, are shown in Fig. 2b. After exposure, the intensity of PL peaks does not vary essentially. But after some period of time (5 ..8 days), transformation of recombination spectra was detected. The intensity of impurity PL increased for GaP by almost twice and for GaAs by almost 1.5 times, respectively. The situation for InP crystals was more complex. The PL intensity oscillates with time, and the behavior of individual bands is not symbate (Fig. 2b).

Since the most efficient action of magnetic field was detected for the magnetic induction of $60 \mathrm{mT}$, this value was used for the further multi-pulse investigations. Fig. 3 presents the results for GaP, GaAs, and InP samples treated in the regime II. One can see that the intensity of GaP PL increases after the treatment, as in the previous cases, but much stronger. The behavior of observed bands for GaAs and InP was also expected. The MF exposure results in small decrease in their emission intensity. The relaxation process of PL bands was observed in further measurements. The synchronous changes appeared until reaching some quasi-steady state. The latter almost did not differ from the initial ones for GaAs and InP crystals, but differed essentially for GaP. The least sensitivity to the given regime of treatment was obtained for GaAs crystals. Apparently, the longerterm regime is needed for this material.

These features of the PL changes of the studied semiconductors could be caused by two competing factors: (i) change of radiative and non-radiative transition probabilities and (ii) change of the concentration of recombination active centers. In our case, essential changes of empirical constants in Eq. (1) were not detected. We consider therefore the second reason to explain the observed features. By analyzing the data obtained, it is possible to draw the following conclusions. There are (i) symbate (Fig. 2a, for GaP during all research, up to 6 and after 9 days for GaAs; Fig. 2b, up to 3 days for all curves; Fig. 3, after treatment for GaP, during all research for GaAs, up to 7 and after 15 days for InP) and (ii) not symbate (Fig. 3, between 1 and 3 days for $\mathrm{GaP}$ and $\mathrm{InP}$, between 3 and 6 days for GaAs, between 8 and 28 days for $\mathrm{InP}$ ) variations of PL intensities. The first of them can be associated with the change of nonradiative recombination channel, and the second one with possible cross transformation of defects that are responsible for observed emission.

Let us try to give a physical explanation to the observed transformations. It is known [11] that WMF treatment can result in migration of non-metallic component of binary semiconductor compound in the bulk. It is possible, in our case, that enriching the surface of the material under study with $\mathrm{Ga}$ atoms was observed (the migration of As atoms into the bulk coincides formally with appearance of more gallium on the surface as compared with the reference case). Likely, due to WMF treatment the destruction of non-equilibrium vacancy-contained complexes occurred. After that, $\mathrm{V}_{\text {As }}$ appeared in the bulk of III-V compounds. These point defects have small lifetimes and can take part in formation of defect complexes. The latter may serve as recombination centers. It seems that the level of mechanical stresses decreases with the increase of the level of structural perfection of multilayer structures. Unfortunately, this state of semiconductor material is thermodynamically unstable, and reverse processes are observed some time after the treatment. In this case, some As atoms are not able to return to their initial state after the WMF treatment. This occurs because they occupy more energy-favorable positions. As a result, there is a tendency for PL spectra to return, in 30 days after the treatment, to the positions only slightly different from the initial ones. After 60 days, almost all PL curves return to their initial positions.

According to $[5,7,12]$, we should believe that the centers of fast (non-radiative) recombination are related to isolated $\mathrm{V}_{\mathrm{Ga}}$ and $\mathrm{V}_{\mathrm{As}}$, background $\mathrm{Fe}$ impurity atoms and dislocations, for GaAs, InP and $\mathrm{GaP}$, respectively. The interaction of WMF with indium phosphide was studied earlier [13], but in that case both the material microstructure and regime of treatment were different. 


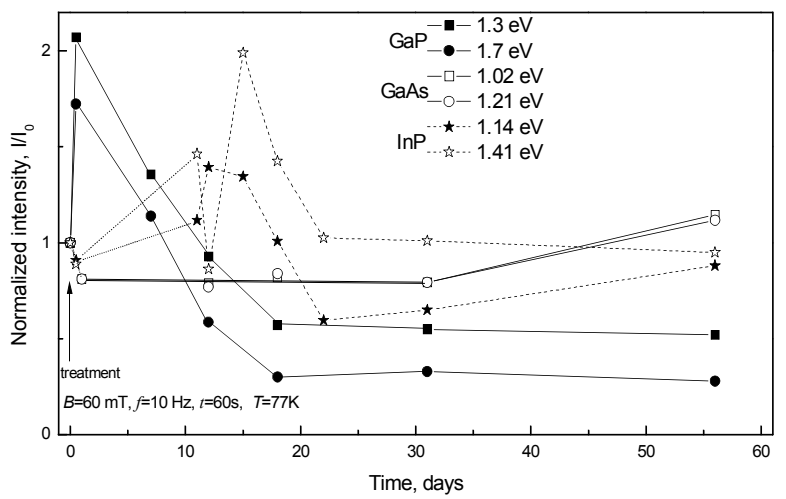

Fig. 3. Intensity of PL bands (at $77 \mathrm{~K}$ ) of III-V crystals as a function of time after MF treatment (regime II).

WMF induced modification studied in $[1,14,15]$ was related to the change of the positions of dislocations in solid state. In those works, a possibility in principle for dislocation motion under WMF action was demonstrated. So, this confirms a possibility of a generation/annihilation of non-radiative centers in gallium phosphide. These processes in GaAs and InP can occur due to migration of the vacancies of non-metal component to the near-surface region [11] due to destruction of V-containing complexes caused by WMF treatment.

Thus, we can discuss the impurity-defect reorganization in semiconductor materials using the modification of their spectroscopic properties. One can propose the following scheme of defect structure modification caused by WMF treatment. First of all, it is destruction of vacancy complexes in the material bulk. Then the diffusion of destruction products (e.g., vacancies) starts. It can take place during the long-term period (up to several months for some semiconductors). First, we have the enrichment of the near-surface region of semiconductor material by III-group component. Later, the reverse process could be observed due to the concentration gradient of As atoms. Due to these transformations in defect structure of the samples studied, the nonmonotonic and long-term changes in spectroscopic characteristics of semiconductor materials are observed. Our results are in a good agreement with the experimental data presented in [4], where similar nonmonotonic processes were detected for another III-V compound (InAs).

The diffusion processes are determined mostly by the initial impurity-defect composition of semiconductor material, doping level and temperature. This explains the difference in PL data for the same material and longterm period of observed transformation. The main issue of this reorganization is the small energy value $\left(\sim 10^{-5} \mathrm{eV}\right)$ obtained in the magnetic field. According to the traditional approach, this energy should be much higher than the energy of thermal fluctuations of electron gas at room temperature. The reliability of these experimental results was repeatedly and carefully studied and confirmed [16-18]. In these works, different mechanisms that can be realized at interaction between solids and WMF were analyzed.

One of these mechanisms is the spin-transformation mechanism (in our experimental conditions, it is most appropriate). The magnetic field is able to change the spin state only in those pairs for which the difference between the energies of singlet and triplet states is comparable with $U_{M}\left(U_{M} \approx g \mu_{\mathrm{B}} B\right.$, with $g$ being the " $g$ factor", and $\mu_{\mathrm{B}}$ the Bohr magneton) [17]. The important requirement of some WMF effects is the presence of paramagnetic impurity as a part of a complex. On the other hand, there is no need in magnetic impurity of such kind in some cases [17]. The most probable impurities in our experiments (background and introduced) were copper, tellurium, oxygen and silicon. Some of them are diamagnetic while others are paramagnetic materials. Obviously, the observable reorganizations are not related to the paramagnetism of impurities. The effect of electromagnetic induction can appear in semiconductor materials at the WMF treatment. Its value can be estimated from the well-known relation $\varepsilon=-\frac{\partial \Phi}{\partial t}$, where $\Phi=\int_{S} B_{n} d S$. For $B=60 \mathrm{mT}, \tau=1.2 \mathrm{~ms}$ and $S \sim$ $1 \mathrm{~cm}^{2}$, we have $\varepsilon \approx 5 \mathrm{mV}$. Obviously, such voltage is not able to induce any structural modifications. However, spin-transformation can determine the reaction of defects destruction. WMF causes reorientation of electron spin. This can result in weakening of chemical bonds and destruction of the complex formed by this atom. As a result, non-equilibrium native point and impurity defects appear, which induces local quasichemical reactions. The next evolution of appeared products causes processes related to long-term transformations of the physical properties of semiconductor material. However, it is impossible to establish reliably the nature and concentrations of structural defects that arise from the mentioned destructions. It is possible to ascertain only that these defects can form the radiative and non-radiative recombination centers that result in the changes of the intensities of observed luminescence bands.

\section{Conclusions}

In summary, the dependences of PL intensity on the time after the WMF treatment of Czochralski-grown III-V semiconductor compounds have been investigated. The results and conclusions of this study can be summarized as follows. (1) The aftereffect is qualitatively the same for all the compounds studied. It consists in the oscillatory changes of PL intensity in the impurity wavelength region to some quasi-equilibrium state not always coinciding with the initial state. (2) The radiative recombination bands related to DA pairs react more efficiently. It was found that PL of GaAs, InP and GaP samples becomes intense after a short-time interval 
(5..7 days) after the treatment. These phenomena indicate that the increase of PL intensity is mainly caused by the WMF-deactivated non-radiative centers. (3) WMF treatment results in the change of the concentration of local centers in III-V compounds that are probably both radiative and non-radiative recombination centers.

The important feature of our results is the sensitivity of semiconductor material to both the singlepulse and multi-pulse treatment. We also noticed that the energy of free electrons is very low to induce the observed transformations. Therefore, we used the spin mechanism to explain the obtained results of experiments. For obtaining quantitative values of observable transformation further researches are needed.

\section{References}

1. Yu.I. Golovin, Magnetoplastic effects in solids // Physics of the Solid State, 46, No 5, p. 789-824 (2004).

2. M.N. Levin, A.V. Tatarintsev, O.A. Kostsova and A.M. Kostsov, Activation of a semiconductor surface by a pulsed magnetic field // Technical Physics, 48, No 10, p. 1304-1306 (2003).

3. M.N. Levin, G.V. Semenova, A.V. Tatarintsev and O.N. Shumskaya, The effect of treatment in a pulsed magnetic field on the kinetics of oxidation of indium phosphide crystals // Technical Physics Letters, 31, No. 9, p. $762-764$ (2005).

4. M.N. Levin, G.V. Semenova, T.P. Sushkova, É.A. Dolgopolova and V.V. Postnikov, The effect of pulsed magnetic fields on the real structure of indium arsenide crystals // Technical Physics Letters, 28, No. 10, p. 818-820 (2002).

5. K.D. Glinchuk, A.V. Prokhorovich, V.E. Rodionov and V.I. Vovnenko, The scheme of electronic transitions via $0.94,1.0,1.2$, and $1.3 \mathrm{eV}$ radiative centres in $n$-GaAs // Phys. Status Solidi (a), 41, No. 2, p. 659-668 (1977).

6. A.B. Georgobiani, A.V. Mikulyonok, I.G. Stoyanova and I.M. Tiginyanu, Non-equilibrium carrier radiative recombination in indium phosphide single crystals // Phys. Status Solidi (a), 80, No. 1, p. 109-115 (1983).
7. S. Doğan, S. Tüzemen, Direct recognition of nonradiative recombination centers in semiinsulating LEC InP:Fe using double excitation photoluminescence // J. Luminescence, 128, No. 2, p. 232-238 (2008).

8. Y.P. Varshni, Temperature dependence of the energy gap in semiconductors // Physica, 34, No. 1, p. 149-154 (1967).

9. S.M. Dishman, D.F. Dalg, W.P. Knox, Deep hole traps in $n$-type liquid encapsulated Czochralski GaP // J. Appl. Phys. 43, No. 11, p. 4693-4705 (1972).

10. A.A. Bergh, P.G.Dean, Light-emitting Diodes. Clarendon Press, Oxford, 1976.

11. M.N. Levin, G.V. Semenova, T.P. Sushkova, V.V. Postnikov and B.L. Agapov. Effect of a pulsed magnetic field on the real solid-solution structure in the Sb-As system // Physics of the Solid State, 45, No. 4, p. 639-642 (2003).

12. V.I. Petrov, G.V. Spivak, M.K. Antoshin, Local cathodoluminescence and its capabilities for the study of band structure in solids // Sov. Phys. Usp. 29, No. 4, p. 364-380 (1986).

13. V.V. Milenin, R.A. Red'ko, Defect reorganization induced by pulsed magnetic field in porous InP // Semiconductor Physics, Quantum Electronics and Optoelectronics, 13, No. 3, p. 330-333 (2010).

14. V.A. Morozov, Plastification of $\mathrm{NaCl}$ crystals under the combined action of short mechanical and magnetic pulses // Physics of the Solid State, 45, No. 10, p. 1932-1934 (2003).

15. Yu.I. Golovin, R.B. Morgunov, S.E. Zhulikov, V.A. Kiperman and D.A. Lopatin, Dislocations used to probe the defect state of an ionic crystal lattice excited by a pulsed magnetic field // Physics of the Solid State, 39, No. 4, p. 554-558 (1997).

16. V.N. Bingi, A.V. Savin, Effects of weak magnetic fields on biological systems: Physical aspects // Phys. Usp. 46, No. 4, p. 259-291 (2003).

17. R.B. Morgunov, Spin micromechanics in the physics of plasticity // Phys. Usp. 47, No. 2, p. 125 147 (2004).

18. Ya.B. Zel'dovich, A.L. Buchachenko, E.L. Frankevich, Magnetic-spin effects in chemistry and molecular physics // Sov. Phys. Usp. 31, No. 5, p. $385-408$ (1988) 